\title{
Radiology Peer Review as a Tool to Improve Healthcare Delivery Is Far From Ideal
}

\author{
David Dinhofer* \\ Advanced Medical Imaging and Informatics, LLC, USA
}

Submission: August 14, 2017; Published: January 09, 2017

*Corresponding author: By David Dinhofer, MD, MSMI, FACR, Advanced Medical Imaging and Informatics, LLC, USA, Email: david.dinhofer@dinhofer.net

\section{Abstract}

Peer review in medicine is the accepted standard for oversight and review of individual physician performance [1]. The system is inherently flawed due to external factors, bias and human fallibility [2]. With changing focus on ethical principles and process improvement techniques in the practice of medicine, we should be able to do better. We need to consider medical ethical principles and transparency with documentation of process improvement to develop and maintain the public trust.

Radiology has been the forerunner in the use of technology in everyday practice. Peer review in radiology has become easy to use with current software [3]. Any time a radiologist reviews an old study, it can be marked with a peer review coding and submitted to any number of programs for indexing and analytics. As it is so easy, the peer review process is in full swing in most institutions. As such, the problems with peer review are also easily observed. In this article I discuss the current problems noted from the perspective of a radiologist and some ideas for improvements in the peer review system using radiology as an example.

\section{Overview}

Peer Review is the currently accepted best practice for oversight and improving physician related work product [4].

It is far from ideal. There are three major issues. At the top of the list is the loss of value due to lack of utility in process improvement. Bias is second and appears to be an insurmountable problem (reference). Lastly, forced participation without obvious beneficence is disingenuous. The radiologist has no reason to do anything more than protect their position in the institution. I believe that most radiologists are focused on the ideal of improving the system. However, it is easy to be jaded when the obvious goal is to punish offenders. In this way, the system encourages individuals to report only positive results.

Malpractice is out of control. The degree to which it affects our ability to improve healthcare is unclear. It is clear that malpractice litigation has a major effect on a physician's ability to confront and correct errors due to routine gag orders during a claim and suit. From an ethical perspective, it is unclear about the real benefit to the patient. It enriches attorneys at the expense of patients and the system. The current justification is that the wronged individual (injured party) deserves their day in court and to be made whole. Yet, how do you make whole an individual that may have lost a limb or important organ? How do you bring back someone to life? Money is the tool we use to compensate someone for an injury or loss. The legal system then adds extra money as punitive damages to satisfy the injured party that someone has been punished. The logic is wrong. Process improvement techniques are a wellestablished way to find and correct errors. Yet, how can we identify problems when the legal system demands that a physician in a legal case not discuss the case for fear of discovery? What does punitive damages do to help the next victim of a medical error? The medical community needs to have the freedom to identify errors without fear in order to come up with corrective actions so that the injury won't be repeated. We may not be able to fix the injured party but we will fix countless others in the future.

The second major problem is that the peer review process is biased and misleading. Bias comes in many different ways. Bias is anything that influences a decision in a non-objective direction. It can be an individual bias toward a diagnosis [2]. It can be an individual's dislike of a test or a person. It can be fear of retribution from someone on the hospital staff or medical director. It can be fear loss of employment. It can be a tool to force someone to leave. It can be a tool to maintain a treatment protocol that an institution needs to be profitable (?Miller ref). Any of these reasons can be a source of bias in the peer review system. Individual bias is almost always present despite even the best individual's efforts to be objective. 
The real value of the peer review system is in its creation. We now have a tool to examine the radiologist work product in a microscopic way. How valuable is this if we can't develop and improve processes for patient care, it is a dead end. As we move toward evidence based medicine, we need to keep in mind that the evidence is in the knowing and understanding of the current process. It is in our ability to analyze objective data and come up with process improvements. It is this standard method that will create the evidence based care practices to be used for future care.

To have a functioning peer review system, the system should be transparent. Details of the deviations should be available for review in order to identify good and bad decisions with an emphasis on improving these decision nodes. Our current peer review process hides details for fear of discovery.

The main function of the physician peer review system is to provide the minimum needed data requirements to produce reports for institutions to use to show they are meeting institutional, federal and medical society standards. It is ethically unsound as his process fails to improve patient care and reduce errors.

The key goals of process improvement are not met when we cannot analyze the process. The dominant peer review system in the US, RADPEERC, depends completely on individual radiologists marking a study [5]. RADPEER doesn't save or collate data of the error. The peer review process creates extra work for physicians and forces them to evaluate colleagues with whom they work without any substantive benefit. Anonymity and lack of accountability encourages abuse. While this is not true for peer review in all institutions, anonymity is encouraged to avoid issues of bias. It fails the principle of beneficence since it fails to demonstrate real change in reducing errors for the reasons above. Additionally, there is no transparency by design.

The ACR created a unified reporting system called, RADPEERC to make it easy for radiologists to meet the requirements of CMS, JCHO, and their individual institutions and designed to meet the institutional, federal and societal guidelines. These guidelines were a direct response to the IOM report on medical errors. In the RADPEER (C) system:

a. Each radiologist is given a unique identifier to allow for anonymization.

b. When a radiologist colleague interprets a study with a prior study, that radiologist can submit a review code to RADPEER(C) for that study which will link it to the original interpreting radiology for the prior study.

c. It is up to the current reviewer to correct errors that are clinically significant (something that is done anyway).

d. However, the current reviewer is also responsible to report any clinically significant misses to a responsible party in the radiology department.

e. A file is created for the each radiologist. f. Errors are stored in the file according to the rating system.

g. If there are similar errors noted in the file, the radiologist is asked to review the subject either individually or at a educationally venue.

h. The radiology group receives a report from RADPEER that lists their performance as compared to the database as well as individual performance statistics.

i. There is no data in the system about what kind of errors are made only the type of study.

\section{Discussion of Ethics Issues and Limitations}

\section{Lack of transparency}

Transparency is supported by respect for autonomy and professional relations (Childress and Beauchamp). There are two reasons for lack of transparency. One is to protect patient health information and the other is to protect the physician's reputation. It is obvious that the physician reputation should not supersede patient's right to information about their health. As noted above, the main factor blocking open discussion is the gag effect of malpractice.

This is the primary problem and road block to developing a process improvement. The ability to openly discuss errors without reprisal is the environment needed to create a fix for the error. This was shown to be a key tool for improving the airlines flight failures [6]. With the estimated number or medical errors well into the 10's of thousands, it is clear that our current system is not working and more drastic steps are needed [7]. Tort reform with a focus on protecting physicians from punitive damages and loss of reputation from individual events is the ultimate mechanism to improve care and diminish errors.

\section{Beneficence [8]}

The main value of peer review is to identify the worst offenders in order to weed them out of the system or mark for retraining. When the system is biased, the results cannot be trusted. Additionally, without transparency, the data needed to review and create a process improvement plan is lacking.

The value of removing bad physicians is high. However, the peer review system fails to help the majority of physicians who may need to minimal corrections in their performance. I put forth that when we add up these small improvements, they will foster a major reduction in errors.

The peer review system is not robust. A system with transparency, attention to detail and open discussion could have far greater value without losing the value of weeding out bad actors. These affects would be a greater beneficence.

\section{Maleficence}

The inability to identify and create process improvement for all players puts patient at risk. This delays creation or process 
improvement plans causing harm to patients. Sharing recurring errors and process improvements is lost harming many more patients. Indeed, bias may hide bad physicians.

\section{Protecting patient health information (PHI)}

Patient health information privacy is a concern in a transparent system. Anonymization of data is not done well and remains a concern (Sweeney). These concerns need further evaluation and discussion. A continuing dispute over whether the rights of society to medical improvement outweighs the right of the individual to privacy needs to be answered. Many individuals are willing to share their PHI in an effort to improve the system. This volunteerism may be the key to success of a transparent system without removing the right to privacy. The medical community often balances maleficence and beneficence. Consider an operative procedure to cure cancer. The patient is often disfigured and put through a traumatic procedure for the possibility of a cure. We should look at the loss of the malpractice liability as a surgical cure for the system. This is not to say that individuals shouldn't be cared for or compensated due to the medical error. The individual deserves compensation in a way to ease the error made. This should be done so as to remove the financial gain of attorneys. This will make more money available for compensation and allow the system to accommodate and anticipate costs.

\section{Patient centered care}

The ultimate goal is to improve patient care for as many as possible and reduce the time for a process improvement to be initiated. In a truly transparent system, peer review becomes obsolete. All the data is available for review and analysis. True patient centered care focuses on using whatever is at our disposal to improve the patient's health outcome without bias. Until such time, that this is possible, we need to consider that the peer review system is a necessary evil.

\section{Sources of bias in peer review:}

i. $\quad$ Outside pressures to keep a hospital contract

ii. Medical legal issues will force peer review to be supportive of the individual in a group in order to safe guard the groups standing in a hospital unless the error is so bad that even a non-radiologist can identify the error.

iii. This limits the purpose of peer review to only big issues when little issues are key to process improvement.

iv. Besides this, medical legal issues force silence of the accused to protect their defense in a suit. In addition, the legal bias of building a case will often force a physician to fight a non-defensible position in order to lower a settlement. This colors the issues and often obscures the real error and issues surrounding the error.

v. Pressures on radiologists to do more (faster TAT's and increased volume) adds pressure on radiologists to take the peer process less seriously and cut corners. The main way this happens is by limiting the peer analysis to positive cases.

\section{Does peer review meet the patient centered ethical approach?}

Keys issues of the patient centered ethical standard include patient choice, satisfaction and safety. Patient choice is based on patient's preferences and need for knowledge creating an environment where patients can make a choice. Patient satisfaction is broad and often hidden in radiology as they are usually unaware of the details of imaging practices. In general, they want access to imaging services, affordable pricing, timely access and reporting, confidences in the services and ability to seek second opinions. Safety is non-descript for the patient. Radiation is the biggest fear but the least important issue. The most important safety issues are more mundane including the usual: wrong test ordered, wrong patient, wrong side, incorrect diagnosis, missing report, misunderstanding of the referring physician, etc. Of course, the balance of benefit to risk must be in favor of the patient and decided by the patient.

\section{Evidence based medicine in Radiology}

Radiology is failing to lead in this important area. The ACR has made a great effort in creating appropriateness criteria and practice guidelines. However, the amount of the information is overwhelming for the individual radiologist; remember, a picture is worth a thousand words. This is an underestimate of the information in a radiology exam. Too often, we limit our report to an overall interpretation. Change is inevitable. The radiologist is image oriented whereas practice guidelines are in print. Image based reporting systems need to be created to fill this void. Currently a few are out there. Usual areas of problems in evidence based medicine exist, mainly, one method doesn't fit situations. Most cases are straight forward. Consolidative pneumonia shows as a white in an area that is usually dark grey and air bronchograms can be seen in the white area. Major fractures can often be seen from 10 feet away. Other findings can be more subtle. When do we say bowel wall is thick due to disease or due to bowel contraction? What is the proper anterior fat pad sign in the elbow? Why should we take an image of the elbow when we see a fracture of the ulnar with no other findings on the wide wrist view? These are specific issues where a robust easy-to-use evidence database is needed.

Questions for building an evidence based system

1. How can we build an evidence based system for imaging diagnosis?

2. How can we make evidence based medicine easy to use in radiology?

3. How would we create an evidence based system in Radiology that can build on itself?

4. Can we show the value of this system and gain acceptance by radiologists, referring physicians and the public?

\section{Considerations for an ideal gold standard for improving patient care in radiology and medicine}

What is the ideal? Should we use all available information and tools at our disposal? These include: a modified peer review, 
patient tracking and follow up, random case review and follow up, AI guided case selection and analysis, and evidenced based processes with analysis of outcomes.

\section{Considerations of what is practical?}

We need to focus our efforts on what is practical and achievable. We should continue our efforts in tort reform. A growing movement to create single payer system for medical care may supersede tort reform and force changes in medical liability as the state becomes the major purveyor of health care.

Maintaining timeliness is key. Any system would have to have a rapid response. AI is the only practical tool for rapid response. Computer aided diagnosis is not practical or widely available. Education is still a mainstay of maintaining quality. Any secondary system should be predominantly a teaching tool (Morbidity and mortality conferences) in which the cases are reviewed (where secondary is any system that doesn't use direct data analysis). Use internal institutional data systems with creation of identifiers as markers for errors or warning system. Creation of deep learning systems to aid in early warning of errors or problems. $+\mathrm{A}$ distributed patient record controlled by the patient with access granted by the patient is being considered and promoted by some entities [9].

\section{Recommendations}

1. Peer review, currently, is only succeeding in identifying the worst offenders. Peer review can be improved by focusing on removing as much bias as possible. This can be accomplished by utilizing independent reviewers such as individuals or groups outside of the of the reviewed practice area and routine exclusion (friends, family and other known relations). Independent reviewers can be a professional group dedicated to peer review or a pool of independent groups that are geographically separate and independently managed possible by a professional medical society (the ACR could serve this function for radiology).

2. Tort reform remains key to open discussion and transparency.

3. Development of a new gold standard which has access to anonymized PHI. This would include AI and Deep Learning tools. The goal being rapid identification of errors and notification before a clinically significant error occurs.

4. Maintenance of health care teams made of physicians, nurses, patients and others involved in patient care working together offers the best current solution with its checks and balances.

5. No impediments to having a review of any disputed case.

6. Continued use of morbidity and mortality conference as an inhouse educational tool which should be a source of CME or mandated as special CME with and protected as much as possible from discovery.

7. Medical societies should actively promote to the public and their legislators tort reform to create an environment conducive to open discussions to improve patient care.

8. Transparent discussion and information so the public will be able to see all issues related to their care openly which will promote trust and improve care

\section{Conclusion}

The peer review system is far from perfect but remains a necessary evil until the public can accept that malpractice litigation is not the best way to improve patient care. True process improvement will come when the medical community can openly and transparently review the source of errors. With tort reform and improvements in the information retrieval, data analytics will be the main tool for process improvement utilizing peer discussions. Peer review in health care delivery along with morbidity and mortality conferences will continue to be an important educational tool and a way to consider the method for improvement.

\section{References}

1. Mahgerefteh S, Kruskal JB, Yam CS, Blachar A, Sosna J (2009) Peer Review in Diagnostic Radiology: Current State and a Vision for the Future 1. Radiographics 29(5): 1221-1231.

2. Croskerry P (2002) Achieving quality in clinical decision making: cognitive strategies and detection of bias. Acad Emerg Med 9(11): 1184-204

3. Abujudeh H, Pyatt RS, Bruno MA, Chetlen AL, Buck D, et al. (2014) RADPEER peer review: relevance, use, concerns, challenges, and direction forward. J Am Coll Radiol 11(9): 899-904.

4. Swensen SJ, Johnson CD (2005) Radiologic quality and safety: mapping value into radiology. J Am Coll Radiol 2(12): 992-1000.

5. Jackson VP, Cushing T, Abujudeh HH, Borgstede JP, Chin KW, et al. (2009) RADPEER scoring white paper. J Am Coll Radiol 6(1): 21-25.

6. Larson DB, Nance JJ (2011) Rethinking peer review: what aviation can teach radiology about performance improvement. Radiology 259(3): 626-632.

7. Kohn LT, Corrigan JM, Donaldson MS (2000) To Err is Human: Building a Safer Health System. Institute of Medicine, National Academies Press, Washington (DC), Newyork, USA, p. 112.

8. Childress JF, Beauchamp TL (2001) Principles of biomedical ethics. Oxford University Press, USA.

9. Abiteboul S, Alexe B, Benjelloun O, Cautis B, Fundulaki I, et al. (2004) An electronic patient record" on steroids": distributed, peer-to-peer, secure and privacy-conscious. Volume 30, Proceedings of the Thirtieth international conference on Very large data bases, VLDB Endowment, Toronto, Canada. 
This work is licensed under Creative Commons Attribution 4.0 Licens

DOI: 10.19080/AJPN.2018.06.555740

\section{Your next submission with Juniper Publishers will reach you the below assets}

- Quality Editorial service

- Swift Peer Review

- Reprints availability

- E-prints Service

- Manuscript Podcast for convenient understanding

- Global attainment for your research

- Manuscript accessibility in different formats

( Pdf, E-pub, Full Text, Audio)

- Unceasing customer service

Track the below URL for one-step submission https://juniperpublishers.com/online-submission.php 